\title{
Visual adaptation in Lake Victoria cichlid fishes: depth-related variation of color and scotopic opsins in species from sand/mud bottoms
}

Yohey Terai ${ }^{1,2^{*}}$ D, Ryutaro Miyagi ${ }^{2}$, Mitsuto Aibara ${ }^{2}$, Shinji Mizoiri ${ }^{2}$, Hiroo Imai ${ }^{3}$, Takashi Okitsu ${ }^{4}$, Akimori Wada ${ }^{4}$, Shiho Takahashi-Kariyazono ${ }^{1}$, Akie Sato ${ }^{5}$, Herbert Tichy ${ }^{6}$, Hillary D. J. Mrosso ${ }^{7}$, Semvua I. Mzighani ${ }^{2,7}$

and Norihiro Okada ${ }^{2,8,9^{*}}$

\begin{abstract}
Background: For Lake Victoria cichlid species inhabiting rocky substrates with differing light regimes, it has been proposed that adaptation of the long-wavelength-sensitive (LWS) opsin gene triggered speciation by sensory drive through color signal divergence. The extensive and continuous sand/mud substrates are also species-rich, and a correlation between male nuptial coloration and the absorption of LWS pigments has been reported. However, the factors driving genetic and functional diversity of LWS pigments in sand/mud habitats are still unresolved.

Results: To address this issue, nucleotide sequences of eight opsin genes were compared in ten Lake Victoria cichlid species collected from sand/mud bottoms. Among eight opsins, the LWS and rod-opsin (RH1) alleles were diversified and one particular allele was dominant or fixed in each species. Natural selection has acted on and fixed LWS alleles in each species. The functions of LWS and RH1 alleles were measured by absorption of reconstituted A1- and A2-derived visual pigments. The absorption of pigments from $\mathrm{RH} 1$ alleles most common in deep water were largely shifted toward red, whereas those of LWS alleles were largely shifted toward blue in both A1 and A2 pigments. In both RH1 and LWS pigments, A2-derived pigments were closer to the dominant light in deep water, suggesting the possibility of the adaptation of A2-derived pigments to depth-dependent light regimes.

Conclusions: The RH1 and LWS sequences may be diversified for adaptation of A2-derived pigments to different light environments in sand/mud substrates. Diversification of the LWS alleles may have originally taken place in riverine environments, with a new mutation occurring subsequently in Lake Victoria.
\end{abstract}

Keywords: Environmental heterogeneity, Color vision, Scotopic vision, Visual adaptation, Cichlid, Lake Victoria

\section{Background}

Lake Victoria harbors more than 500 endemic cichlid species $[1,2]$. They are thought to have undergone explosive adaptive radiation during a very short evolutionary period, because Lake Victoria dried up at the end of the Pleistocene and was refilled only 15,000 years ago

\footnotetext{
* Correspondence: terai_yohei@soken.ac.jp; okadanorihiro@gmail.com 'Department of Evolutionary Studies of Biosystems, SOKENDAI (The Graduate University for Advanced Studies), Shonan Village, Hayama, Kanagawa 240-0193, Japan

${ }^{2}$ Graduate School of Bioscience and Biotechnology, Tokyo Institute of Technology, 4259 Nagatsuta-cho, Midori-ku, Yokohama 226-8501, Japan Full list of author information is available at the end of the article
}

[3]. Lake Victoria cichlid species share polymorphic nucleotide sites [4-7] due to this short radiation period. Nevertheless, fixed genetic differences were thought to exist between species at loci responsible for the adaptive traits distinguishing the various forms from one another. The long-wavelength-sensitive (LWS) opsin gene has been identified as one such gene possessing fixed differences among species, and this has been interpreted as an adaptation to contrasting light regimes [8].

Vertebrate visual pigments consist of a light-absorbing component, the chromophore, and a protein moiety, the opsin [9]. Spectral sensitivity is determined by the 
chromophore [with 11-cis retinal (A1-) or 11-cis 3dehydroretinal (A2-derived retinal)] and by the interaction of the chromophore with the amino acid residues that coat the retinal-binding pocket of the opsin in which the chromophore lies [10]. The replacement of A1- with A2-derived retinal in the pigments shifts the absorption to a longer wavelength [11, 12], and the shift is larger in longer wavelength absorbing opsin pigment $[11,12]$.

The visual systems of African cichlids have been studied extensively $[8,13-21]$ because vision is important for food acquisition [22-24] and mate choice [18, 25-27]. African cichlids have eight different opsin genes $[8,13,14,19]$, but only a subset of these is expressed in any individual species [20, 28]. Several Lake Victoria species primarily express the repertoire of four opsin genes [20, 28]: short-wavelengthsensitive opsin gene 2a [SWS2A, $\lambda$ max of pigments with A1-derived retinal (A1 pigments) $=457 \mathrm{~nm}$; $\lambda \max$ of pigments with A2-derived retinal (A2 pigments $)=472 \mathrm{~nm}]$ in single cones; middlewavelength-sensitive opsin genes (RH2A $\beta, \lambda \max$ of A1 pigments $=523 \mathrm{~nm}$ and $\lambda \max$ of A2 pigments $=546 \mathrm{~nm}$ or $\mathrm{RH} 2 \mathrm{~A} \alpha, \lambda \max$ of $\mathrm{A} 1$ pigments $=533 \mathrm{~nm} ; \lambda \max$ of A2 pigments $=555 \mathrm{~nm}$ ) and LWS opsin genes $(\lambda \max$ of A1 pigments $=544-$ $559 \mathrm{~nm} ; \lambda$ max of A2 pigments $=595-611 \mathrm{~nm})$ in double cones for color vision; and rhodopsin (RH1, $\lambda \max$ of A1 pigments $=503 \mathrm{~nm}$; $\lambda \max$ of A2 pigments $=523 \mathrm{~nm})$ in rods for scotopic vision $[17,18,29]$.

The opsins of African cichlids are thought to have adapted to different ambient light conditions varying with depth, water color, and turbidity. The first example described was deep-water adaptation of RH1 pigments in cichlid species from two clear-water lakes, Lake Tanganyika and Lake Malawi. An amino acid change from alanine to serine at position 292 (A292S) shifts the peak wavelength absorbance $(\lambda \max )$ by $11-14 \mathrm{~nm}$ toward blue light. This shift from the longer $(\sim 500 \mathrm{~nm})$ to the shorter $\lambda \max (470-490 \mathrm{~nm})$ is an adaptation from shallow-water to deep-water light regimes [16]. This amino acid change has occurred in parallel in several different lineages [16], and reverse change has also occurred in some of these [30].

In Lake Victoria, LWS genes from species inhabiting rocky substrates have been investigated. Neochromis greenwoodi is distributed along gradients in turbidity. The populations at opposite ends of one gradient fixed different $L W S$ alleles, and these alleles were adaptive to light regimes differing in turbidity [17]. In Pundamilia species, $P$. pundamilia and $P$. nyererei are found in shallower and deeper water, respectively. In each species, the $L W S$ alleles are adapted to the specific ambient light regimes related to water depth [18]. These studies described correlations between adaptive divergence of $L W S$ and male nuptial coloration, suggesting that adaptation of the sensory system to different light regimes drives the divergence of mating signals and leads to reproductive isolation $[17,18]$.

In contrast to rocky substrates, sand/mud bottoms form a continuous environment. Among six species found on sand or mud, the LWS and RH1 alleles have diversified and one particular allele is dominant or fixed in each species [29]. The functions of the $L W S$ alleles are also diversified, whereas those of the $R H 1$ alleles are identical, as shown by absorption measurements of reconstituted visual pigments [29]. A correlation between male nuptial coloration and the absorption of LWS pigments was also observed among six species [29]. However, because the six species were collected from a single locality with low environmental variability, it is still unclear which factors drive genetic and functional diversity of LWS in sand/mud habitats. To address this issue, we compared ten Lake Victoria cichlid species from sand/mud habitats to identify 1) opsin genes with fixed genetic differences, 2) their functional differences, and 3) the adaptive role of the opsin pigments.

\section{Results}

\section{LWS and $R H 1$ were dominated by one allele in each species}

The ten species in this study were distributed from near the surface to the deepest bed $(70 \mathrm{~m})$ in Lake Victoria (Fig. 1, Additional file 1: Figure S1a-j). All species were collected from sand/mud substrates. The sequences of seven color opsin genes (SWS1, SWS $2 A$, $S W S 2 B, R H 2 A \beta, R H 2 A \alpha, R H 2 B$, and $L W S$ ) and one scotopic opsin gene $(R H 1)$ were determined in two individuals from each of the ten species and used to calculate mean distances of synonymous (Ds) and nonsynonymous (Dn) substitutions (Fig. 2a). High values of Dn were observed in $R H 2 A \beta, L W S$, and $R H 1$ (Fig. 2a), indicating amino acid variations in $R H 2 A \beta, L W S$, and $R H 1$. Of the opsins with high Dn values, species-specific alleles were observed from LWS and RH1. Therefore, we further determined the sequences of these two genes (Additional file 2: Figure S2 and Additional file 3: Figure S3) from the ten species (in total, 219 individuals) with different depth distributions (Additional file 1: Figure $\mathrm{S} 1 \mathrm{a}-\mathrm{j}$ ). We divided these sequences into eight $L W S$ allele and six RH1 allele groups based on the amino acid sequences (Fig. $2 b$ and c), because the alleles that differed by synonymous substitution were functionally identical. Thus, the sequences that were in the identical allele groups shared the identical amino acid replacements, as shown in Fig. 2b and c. Several allele groups are described in our previous studies [i.e., H, L, M3, and P [17] 


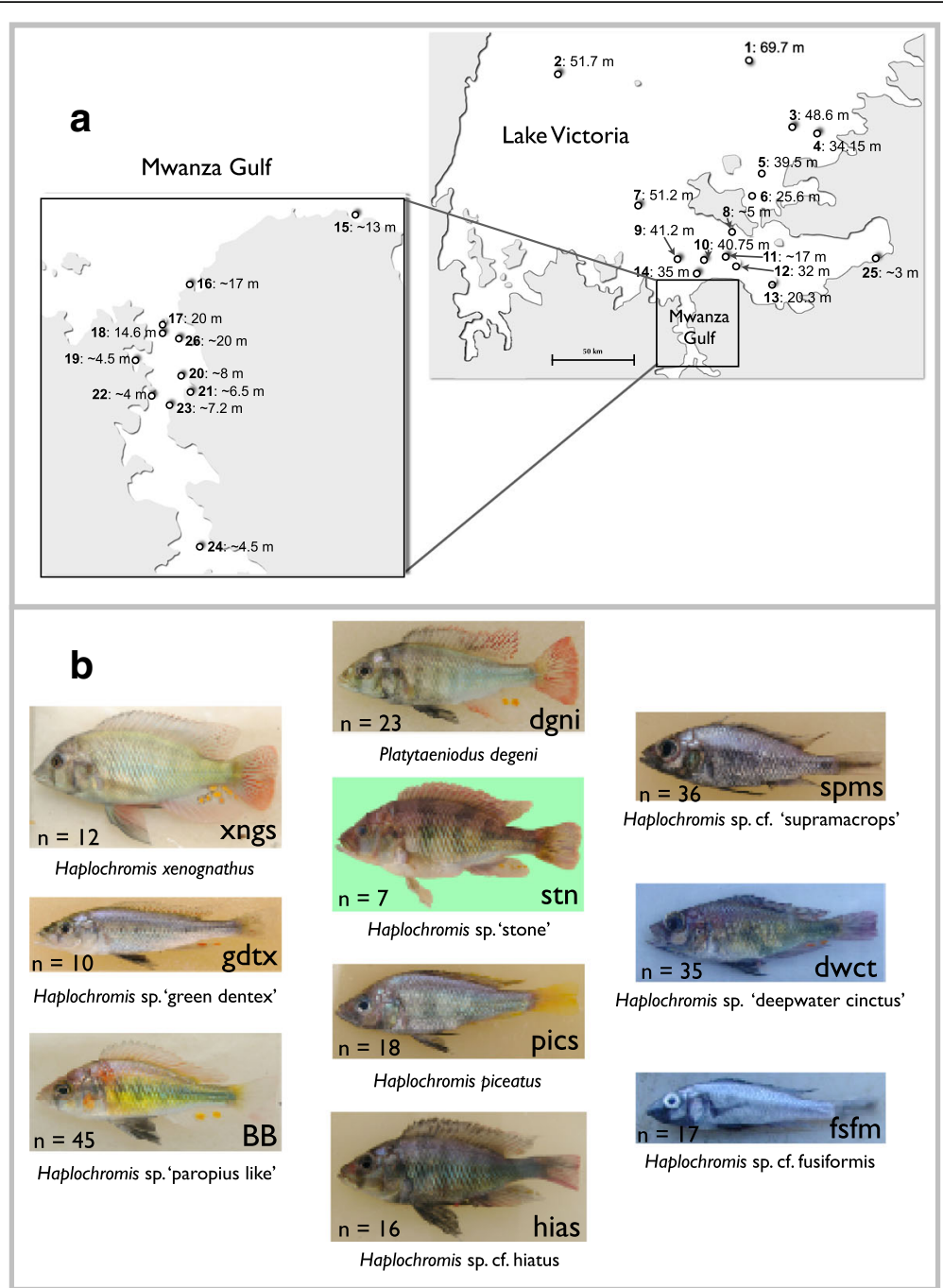

Fig. 1 Sampling localities in Lake Victoria and photographs of cichlid species. a Map of the southern half of Lake Victoria showing the cichlid sampling localities. Numbers indicate collection localities: 1, Offshore Ukara; 2, Offshore Kerebe; 3, Irugwa; 4, Karaju; 5, Usengere; 6, Kweru; 7 , Offshore Lyamwenge; 8, Nansio; 9, Offshore Nyakanyasi; 10, Offshore Makobe; 11, Offshore Mabibi Island; 12, Vessi Island; 13, Offshore Magu Bay; 14, Offshore Makobe; 15, Kayenze; 16, Bwiru Point; 17, Bwiru Point-hippo; 18, Offshore Hippo; 19, Kissenda Bay; 20, South Offshore Chankende; 21, Nyegezi Bay; 22, Nyaruwambu; 23, Nyameruguyu (Python); 24, Offshore Marumbi; 25, Mwabulugu; and 26, Gabalema. b Photographs of cichlid species, with abbreviations for species names and the numbers of individuals used in this study for each species. Fish species order is from shallow- (left-top) to deep-water (right-bottom). The maps were drawn by Y. T. based on original source maps: https://www.google.com/maps. Fish photographs were taken by M. I. and S. M

and Sp, r104V, and r104I [29]]. Based on the allele groups of LWS and RH1, we compared the depth distribution (Fig. 3a) of allele frequencies of LWS (Fig. 3b) and RH1 (Fig. 3c). The abbreviations for species names are shown in Fig. 1b. In the frequencies of $L W S$ and $R H 1$, each species was dominated by one allele (Fig. 3b and c). The dominant alleles in LWS (M3, P, and D; Fig. 3b) and RH1 (r104I; Fig. 3c) varied by depth and were shared by species with similar depth distributions. Based on these results, the distributions of LWS and RH1 alleles were likely correlated with depth, and the adaptation of those alleles was inferred.
In addition to Lake Victoria species, we determined the sequences of LWS from riverine Haplochromis species $(n=8)$.

\section{Functional diversities of RH1 and LWS pigments}

To test whether $L W S$ and RH1 alleles were functionally different, we measured visual pigments that were reconstituted using A1- and A2-derived retinal. The results for several of these measurements were obtained from previous studies $[17,18,29]$. As shown in Fig. 4a-f, we measured newly discovered alleles and an allele $(\mathrm{P})$ that was not measured in the A2 pigments by [18]. We did 


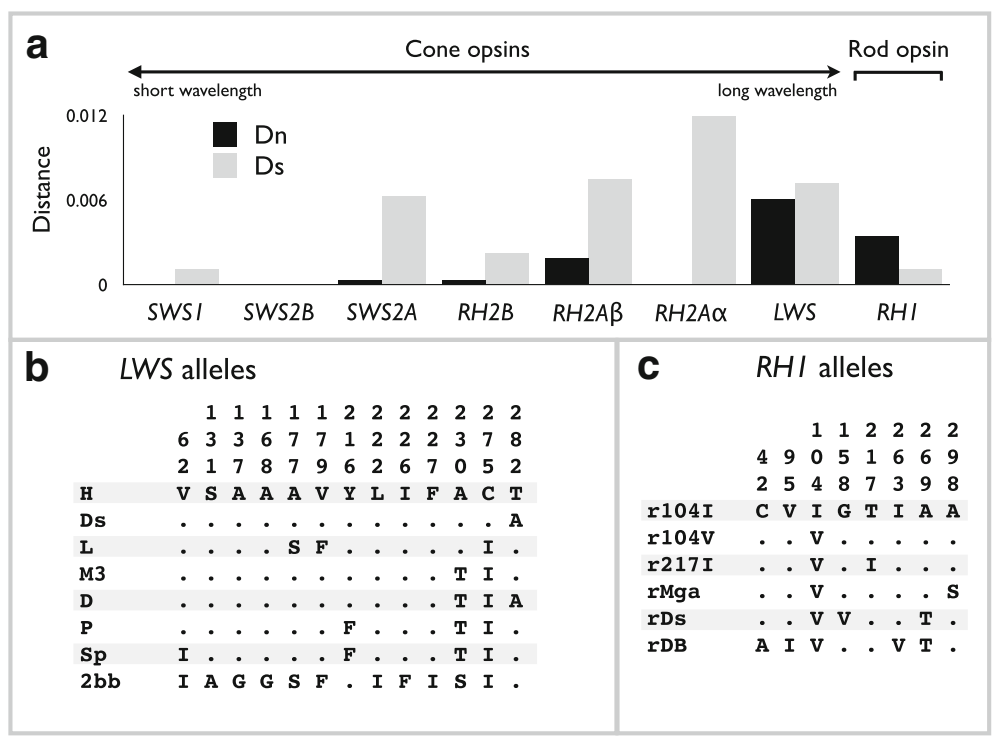

Fig. 2 Diversities of opsin genes in Lake Victoria. a Genetic diversities of opsin genes. Each column indicates mean distance for synonymous (gray) or nonsynonymous (black) substitutions for ten species in Lake Victoria. Amino acid alignments of $\mathbf{b}$ LWS and $\mathbf{c}$ RH1 alleles. Residue positions are numbered according to the sequences of each opsin gene. The amino acid positions 62, 131, 137, 168, 177, 179, 216, 222, 226, 227, 230, 275, and 282 in LWS correspond to 49, 118, 124, 155, 164, 166, 203, 209, 213, 214, 217, 262, and 269 in bovine RH1. The amino acid positions in cichlid $\mathrm{RH} 1$ are identical in bovine $\mathrm{RH} 1$. The dots and letters indicate identical and different residues, respectively, compared with the top line

not obtain the absorption of pigments from $2 \mathrm{BB}$ and r217I alleles (Fig. 3b and c) because of the instability of those pigments. The absorption spectra of the pigments were represented by the peak values $(\lambda \max )$, as shown in Fig. $4 \mathrm{a}-\mathrm{f}$, with the functional differences of the alleles summarized in Fig. 4g and h. For $R H 1$ alleles, the $\lambda \max$ values of $\mathrm{A} 1$ and $\mathrm{A} 2$ pigments ranged from 502 to $515 \mathrm{~nm}$ and from 522 to $539 \mathrm{~nm}$, respectively (Fig. $4 \mathrm{~g}$ and $\mathrm{h}$ ). The pigments from $\mathrm{rDb}$ and $\mathrm{rDs}$ alleles were largely shifted toward red (511 and $515 \mathrm{~nm}$ for A1 and 536 and $539 \mathrm{~nm}$ for A2 pigments, respectively; Fig. $4 \mathrm{~g}$ and $\mathrm{h}$ ). For $L W S$ alleles, the ranges of $\lambda \max$ values of $\mathrm{A} 1$ and A2 pigments varied widely from 537 to $559 \mathrm{~nm}$ and from 568 to $611 \mathrm{~nm}$, respectively (Fig. $4 \mathrm{~g}$ and h). Notably, these wide ranges of $\lambda$ max values resulted from the replacement of only a small number of amino acids (from $\mathrm{H}$ to $\mathrm{Sp}$ alleles in Fig. 2b). For example, the A2 pigments from Ds and D alleles were shifted 29 and $36 \mathrm{~nm}$ toward blue compared with that of the $H$ allele by only one and three replacements, respectively (Figs. 2b and 4h). Hence, the functional diversities of RH1 and LWS pigments were generated by a small number of replacements among alleles.

To reveal the driver for the diversity of $L W S$ alleles, we performed HKA tests for heterogeneity between regions (LWS gene and up- and downstream regions, shown in Fig. 5a) using the ratio of polymorphism to divergence [31]. Based on the LWS allele phylogenetic tree constructed for the $L W S$ gene and the flanking regions (5 kb upstream, LWS gene without exons, and $3.5 \mathrm{~kb}$ downstream, totaling $9492 \mathrm{bp}$ ), we used the $\mathrm{Sp}$ allele as the out-group for HKA tests (Fig. $5 \mathrm{~b}$ ). The contrast in divergence between the $L W S$ gene region (differentiated) and that in the flanking sequences (undifferentiated) was statistically significant (Table 1), with the exception of the $\mathrm{D}$ allele because of a small number of polymorphic sites (no site and six sites in up- and downstream regions, respectively). Based on these results, natural selection has acted on the divergence of the LWS gene to generate the functional diversity of this gene.

\section{Discussion}

\section{Visual adaptation of color and scotopic opsins in Lake Victoria cichlid species}

Among eight opsins, amino acid variations and speciesspecific alleles were observed from $L W S$ and $R H 1$, indicating no species-specific functional difference in other six opsins. As shown in Fig. 4, we summarized the peak absorption of pigments reconstituted by LWS and RH1 alleles and A1- (Fig. 4g) or A2-derived (Fig. 4h) retinal. The absorption spectra of pigments were measured in this study (RH1: rDb, rDs, rMga; LWS: D, Ds, P-A2 pigment) or previous studies (RH1: r104I, r104V; LWS: H, M3, Sp, P-A1 pigment) [17, 18, 29]. The allele distributions of L (rocky substratum) [17] and $\mathrm{H}$ (sand/mud substratum) [29] in shallow water were described in previous studies.

In RH1, the species distributed in deep water (Fig. 3a) possess red-shifted pigments ( $\mathrm{rDs}$ and $\mathrm{rDb}$ ) in both $\mathrm{A} 1$ - 


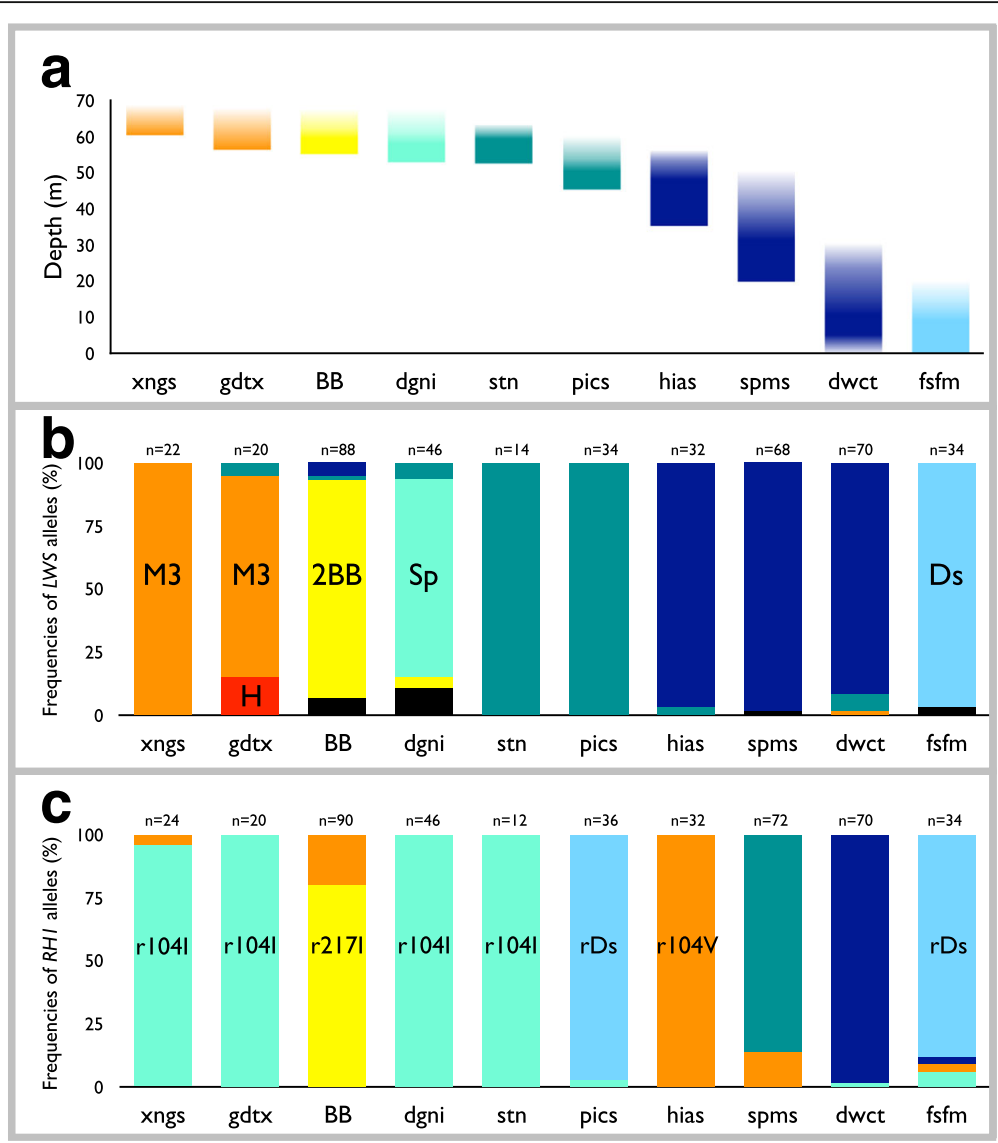

Fig. 3 Cichlid species distributions and the frequencies of LWS and RH1 alleles. a Species distributions by depth are shown in each column. The positions of the columns of species are identical in $(\mathbf{a})-(\mathbf{c})$. Frequencies of $\mathbf{b}$ LWS and $\mathbf{c} R H 1$ alleles in the ten species. The names of the alleles correspond to those in Fig. $2 \mathrm{~b}$ and $\mathrm{c}$, respectively. The number of total alleles per species is shown at the top of the columns. Abbreviations for the species names are shown in Fig. 1b

(Fig. 4g) and A2-derived (Fig. 4h) retinal. In Lake Victoria, the proportion of long wavelengths in the light spectra increases with depth, with the light in deep water dominated by long wavelengths (i.e., $540-600 \mathrm{~nm}$ ) [18, 32]. Thus, the absorption shifts toward dominant light in the deep-water RH1 pigments suggest that the functional changes of RH1 pigments from shallow- to deepwater distribution may be an adaptation for efficient absorption of light wavelength (Fig. $4 \mathrm{~g}$ and $\mathrm{h}$, green arrows). Although the rDs allele predominated in $\mathrm{H}$. pisceatus, this species occurs at shallower depths than the other rDs-predominated species, $H$. sp. cf. fusiformis (Fig. 3). This difference may be explained by the contrasting distributions of these two species. Haplochromis pisceatus and $H$. sp. cf. fusiformis specimens were collected from Mwanza Gulf and offshore, respectively (Additional file 3: Figure S1f and j). Water transparency is higher offshore than in Mwanza Gulf [17], and the water turbidity strongly affects the proportion of long wavelengths in the light spectra $[17,28]$. Thus, the rDs allele of $H$. pisceatus may be an adaptation to the long- wavelength light that rapidly increases with depth in Mwanza Gulf.

In contrast to RH1 pigments, A1- and A2-derived LWS pigments show different aspects. The $\lambda$ max values of LWS pigments reconstituted by alleles predominant in species from shallow to deep water (Fig. 3a) gradually shifted toward blue in both A1- (Fig. 4g) and A2-derived (Fig. 4h) retinal. In the case of A1-derived retinal, the $\lambda$ max values from deep-water species separated from the dominant light in deep water (Fig. 4g, orange arrow), whereas they overlapped with the dominant light in deep water (Fig. 4h, orange arrow) in A2-derived retinal. This suggests that the deep-water A2-derived LWS pigments may adapt to the deep-water light regime, even though the A1/A2 ratio (chromophore usage) of deep water species has not been measured.

In contrast to deep-water, water transmits broad spectra of light (i.e., $400-650 \mathrm{~nm}$ ) $[18,32]$ in shallow-water. The $\lambda$ max values of RH1 pigments reconstituted by alleles predominant in shallow species were located in the middle of wavelength of light, whereas those of LWS A2 


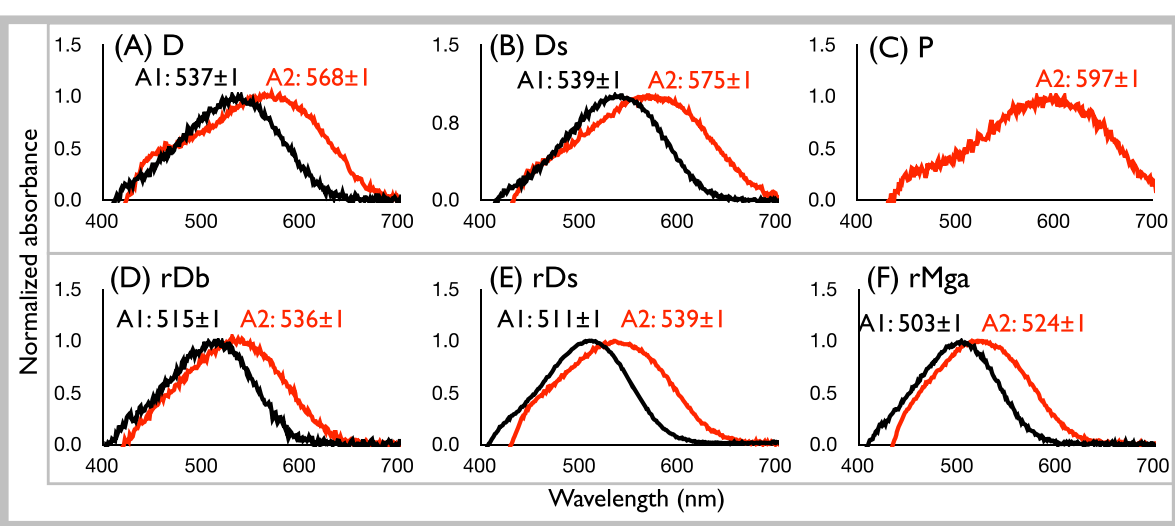

(G) RHI alleles

LWS alleles
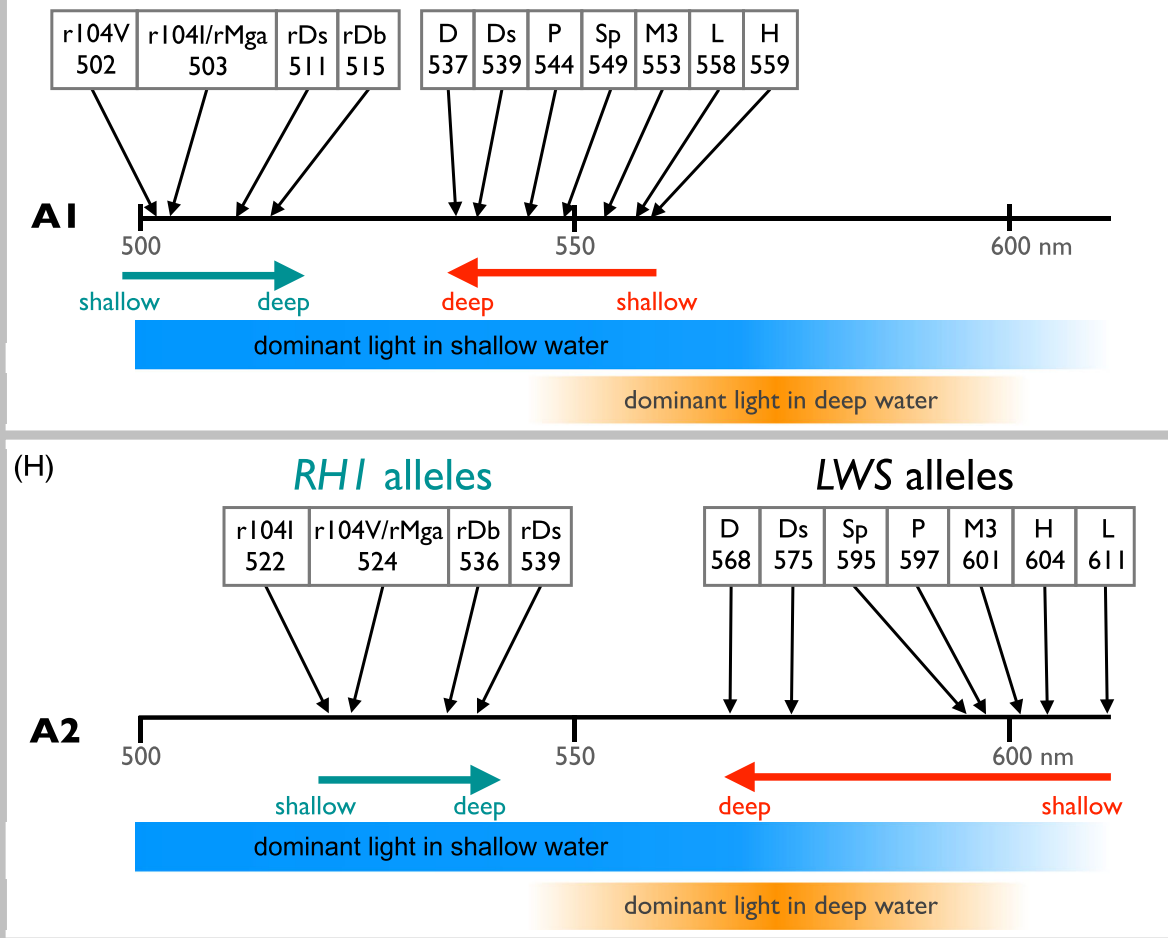

Fig. 4 Functional diversities and adaptation of opsin genes in Lake Victoria. a-f Dark-light difference in spectra of visual pigments. Visual pigments were reconstituted from three LWS alleles [(a), D; (b), Ds; and (c), P] and three RH1 alleles [(d), rDb; (e), rDs; and (f), rMga], with A1(black) and A2-derived (red) retinal. The A1 pigments of the P allele were measured previously [18]. $\mathbf{g}$ Summary of the functional diversities of LWS and RH1 alleles reconstituted by A1-(g) and A2-derived (h) retinal. The $\lambda$ max values and allele distributions of $L$ (rocky substratum) [17] and $\mathrm{H}$ (sand/mud substratum) [29] were described in previous studies. The distributions of RH1 and LWS alleles are shown by green and orange arrows, respectively. The dominant light wavelength in shallow and deep water $[18,28,32]$ is shown by the orange bar

pigments were nearly the end of long wavelength light (Fig. 4h). The absorption of LWS A2 pigments may be an adaptation for color discrimination in broad spectra of light in shallow-water.

The A2 chromophore usage was measured $[16,17]$ and also estimated from MSP data [29] in our previous studies. In our work, the retinal was extracted from the entire eyecup, and the extraction and measurements were performed under conditions in which the unstable A2 retinal could easily degrade. Therefore, the estimation of chromophore usage was more accurate than that determined from measurements of the amount of A2-derived retinal, and we estimated A2 usage from previously described MSP data [20,33]. The estimated A2 proportion was $20-84 \%$ in RH1 and $30-100 \%$ in LWS pigments (Additional file 4: Table S1). This suggests that the proportion of A2 was relatively high in Lake Victoria cichlids, and supports the adaptation of A2-derived LWS pigments. The cichlid fish used in the studies of MSP measurements were laboratory-reared fish [20,33]. In freshwater fish, 


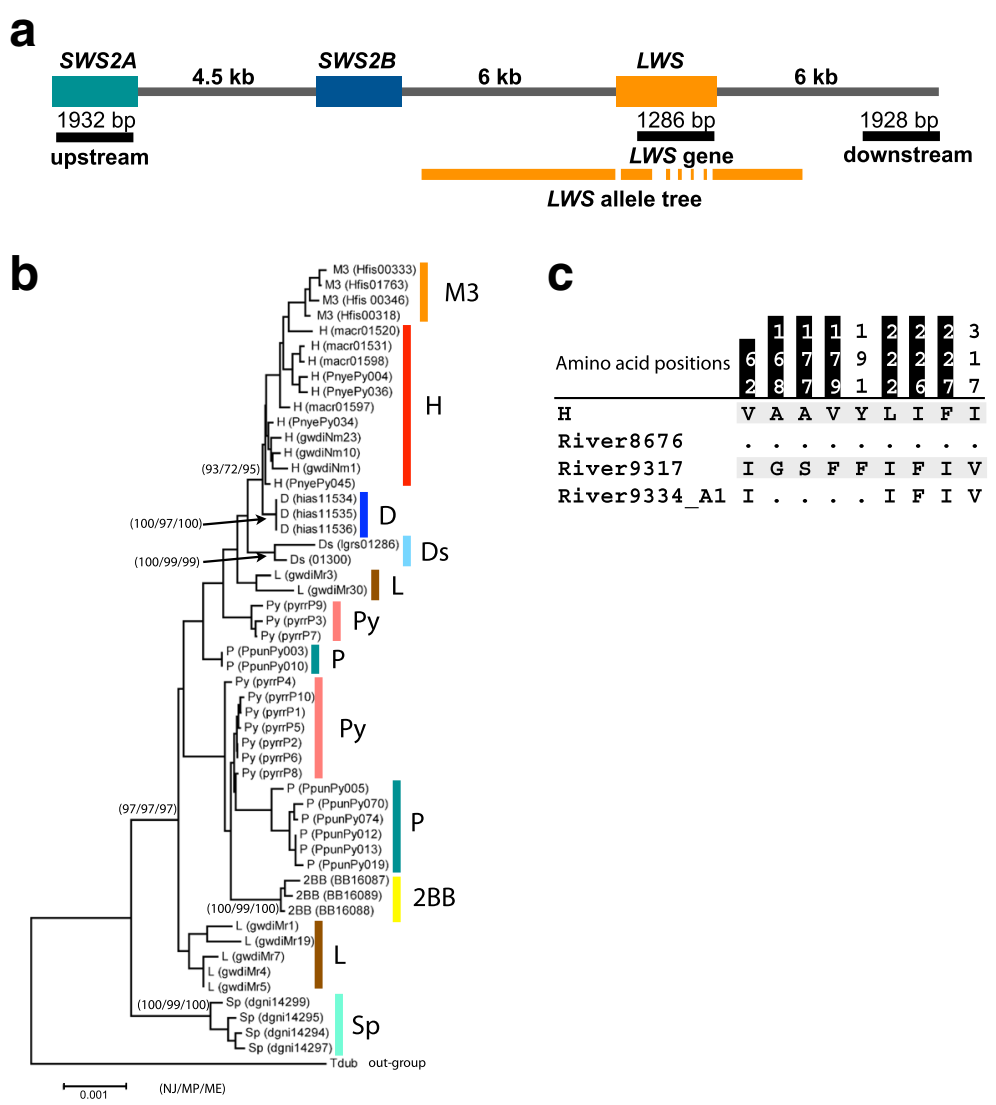

Fig. 5 The evolution of LWS alleles. a The regions used for HKA tests and construction of a LWS allele tree are shown by black and orange lines under the genome structure of LWS and flanking regions, respectively. b Neighbor-joining tree constructed using sequences 5 kbp upstream of LWS, LWS gene excluding exons, and 3.5 kbp downstream of LWS. Bootstrap values are shown for the neighbor-joining tree (left), maximumparsimony tree (center), and maximum-likelihood tree (right) when the values were 95 or more in any tree. The alleles of LWS are shown at the ends of the tree branches. c An amino acid alignment of LWS from riverine species. Residue positions are numbered according to the sequences of LWS. The dots and letters indicate identical and different residues, respectively, compared with the top line. The amino acid positions with differences that are found among the alleles from Lake Victoria species are highlighted in black. The LWS sequences were determined from four riverine species: H. sp. 'katonga' $(n=2)$, H. sp. 'katavi' $(n=1)$, H. sp. 'kitilda-rukwa' $(n=2)$, and H. sp. 'muzu-rukwa' $(n=3)$. An alignment of LWS sequences from riverine individuals and sampling localities are shown in Additional file 5: Fig. S4

chromophore usage can change from A1 retinal in bright light to A2 retinal in dark condition [34]. Therefore, our estimation may include the possibility of under estimation of the A2 proportion. Recently, the coexpression of different opsin genes in a single photoreceptor cell was reported $[35,36]$. In the case of MSP data for LWS

Table 1 HKA test for statistical significance (p) of heterogeneity between regions

\begin{tabular}{llll}
\hline LWS alleles & Up (SWS2A)-gene & gene-down & Up (SWS2A)-down \\
\hline 2BB & $0.0154^{*}$ & 0.7876 & 0.2974 \\
$D$ & 0.285 & 0.2601 & 0.0995 \\
DS & $0.0016^{* *}$ & $0.0047^{* *}$ & 0.6887 \\
M3 & $0.0200^{*}$ & $0.0127^{*}$ & 0.8907 \\
$P$ & $0.0287^{*}$ & $0.0066^{* *}$ & 0.5814 \\
${ }^{*} P<0.05 ;{ }^{* *} P<0.01$ & &
\end{tabular}

pigments, the coexpression of $L W S$ and $R H 2 A \alpha$ ( $\lambda$ max of A2 pigments $=555 \mathrm{~nm}$ ) might be able to generate a similar curve to the curve of LWS pigments with A1/A2 mixture. In this case, the MSP data can be explained by LWS and $\mathrm{RH} 2 \mathrm{~A} \alpha$ pigments with high proportion of $\mathrm{A} 2$ retinal. In RH1, A2-derived retinal usage may also be adaptive, because the $\lambda \max$ values of red-shifted A2-pigments were closer to the dominant light in deep water than for A1derived pigment. Although the chromophore usage has not been determined, the RH1 and LWS sequences may be diversified for adaptation to different light environments in sand/mud substrates. Measurements of A1/A2 ratio using properly-preserved eye samples to avoid A2 retinal degradation from wild-caught individuals of deep water species will reveal the adaptation of A2-derived opsin pigments to deep water light environments in near future. 
The replacement of A1- with A2-derived retinal in the LWS pigments shifted the $\lambda$ max values to longer wavelength range from $31 \mathrm{~nm}$ (D allele) to $53 \mathrm{~nm}$ (P and L alleles). Similar to the variation in absorption spectra with the same chromophore (A1- or A2-derived), the variation of $\lambda \max$ value shift by replacement of A1- with A2-derived retinal was also generated by a small number of replacements among alleles. Although we could not find a correlation between amino acid replacements and $\lambda \max$ value shift by replacement of chromophore, mutagenesis, reconstruction, and measurement of visual pigment absorption spectra will reveal the effect of each amino acid replacement in future.

\section{Evolution of LWS alleles in Lake Victoria cichlids}

Although Lake Victoria cichlid species show little genetic differentiation $[37,38]$, the $L W S$ sequences were highly diversified (Fig. 2) [8, 17, 18, 29]. The LWS sequences from Lake Victoria cichlids were separated into two clades, clade I and clade II, in the phylogenetic tree [8]. However, this tree may not represent the evolutionary process of the LWS gene, because the effects of selection cause parallel substitutions in the coding region of this gene. Therefore, we used the up- and downstream flanking regions and intron sequences to construct a phylogenetic tree of $L W S$ alleles. We used one clade II allele (2BB) and eight clade I alleles (H, L, M3, P, Py, Sp, D, and Ds) in the analysis. As shown in Fig. 5b, the clade II allele (2BB) formed a clade and diverged from the internal clade of clade I alleles. This suggests that the clade II allele is the derived-type allele. In clade I, the alleles from D and Ds formed each clade, respectively. These alleles possessed alanine at position 282 (282A) that corresponds to position 269 in bovine RH1, and this amino acid caused a large spectral shift toward blue. An alanine at this position has never been found in African cichlids except for these two alleles, raising the possibility that $282 \mathrm{~A}$ was generated by a new mutation in Lake Victoria. Indeed, the up- and downstream region sequences among three individuals possessing the $\mathrm{D}$ allele were identical, suggesting that $\mathrm{D}$ is a young allele.

Three deep water species possessed the D allele. This might be explained by either the selection on the D allele in different lineages independently from the ancestral polymorphic alleles, or the selection on the $\mathrm{D}$ allele in the common ancestral lineage of three deep water species. The phylogenetic relationship among Lake Victoria cichlids could answer this question. At present, RAD-tag sequencing is the most powerful tool to reconstruct a phylogenetic tree of closely related cichlid species. RAD-tag sequencing was used in a previous study to examine Lake Victoria rock cichlids [7], but only two species in sand/mud substrates were included [39]. Instead of RAD-tag sequencing, we calculated $F_{S T}$ values between each deep water species and the other species using mitochondrial DNA control region sequences among species used in this study. Each of the species pairs between deep water species with D allele and nondeep water species showed the lowest $\mathrm{F}_{\mathrm{ST}}$ values as follows: $H$. sp. cf. hiatus- $H$. sp. 'paropius like' $\left(\mathrm{F}_{\mathrm{ST}}=0.01\right)$; $H$. sp. cf. 'supramacrops'-Haplochromis piceatus $\left(\mathrm{F}_{\mathrm{ST}}=0.05\right)$; and $H$. sp. 'deepwater cinctus'- H. xenognathus $\left(\mathrm{F}_{\mathrm{ST}}=0.04\right)$. This result suggests that three deep water species may not be close relatives, and it was more likely that the selection might have acted on the D allele in different lineages, independently.

By contrast to young alleles, both clade I and II alleles were found outside of Lake Victoria. As shown in Fig. 5c, the allele composition was one clade I allele (River8676) and one clade II allele (River9317), and one recombinant of both sequences (River9334_A1) (Additional file 5: Figure S4C). Among these alleles, seven out of nine amino acid differences were also found among the alleles from Lake Victoria species (Fig. 5c, highlighted in black). These results suggest that the genetic diversity of $L W S$ has a riverine origin. Hence, the LWS alleles were diversified by using old mutations occurring outside of Lake Victoria and also a new mutation in the lake. The combination of mutations generated by recombination among founder alleles may have been shaped by selection for adaptation to various light regimes in Lake Victoria.

\section{Conclusions}

In this study, we analyzed the nucleotide sequences of eight opsin genes in ten Lake Victoria cichlid species collected from sand/mud bottoms, and showed that the $L W S$ and RH1 alleles were diversified and one particular allele was dominant or fixed in each species. The $L W S$ alleles were fixed in each species by natural selection. The absorption of pigments from RH1 and $L W S$ alleles were largely shifted toward different directions, red and blue respectively, in both A1- and A2-derived pigments. In both RH1 and LWS pigments, A2-derived pigments were closer to the dominant light in deep water, suggesting the possibility of the adaptation of A2-derived pigments to depth-dependent light regimes. The usage of A2-derived retinal in freshwater fish has been well known [40], while only a few studies have payed attention and measured the absorption of A2-derived opsin pigments [17, 29]. In this study, we extensively compared the functional differences between A1- and A2derived pigments in $\mathrm{RH} 1$ and LWS, and demonstrated the importance of A2-derived pigments for adaptation to light environments in freshwater fish.

In addition to a correlation between male nuptial coloration and the absorption of LWS pigments in the 
extensive and continuous sand/mud substrates [29], we revealed that the LWS sequences were diversified for adaptation by using old mutations occurring outside of Lake Victoria and also a new mutation in the lake. These visual sensor adaptation and correlation with mating signal (male nuptial coloration) might be a signature of speciation by sensory drive $[17,18]$, and further studies of the correlation between nuptial colors and visual adaptation will reveal the mechanism of speciation in Lake Victoria cichlid species from sand/mud habitats in the future.

\section{Methods}

\section{Samples}

The Institutional Animal Care and Use Committee of Tokyo Institute of Technology approved the animal protocols and procedures. Ten Lake Victoria cichlid species were examined: Haplochromis piceatus Greenwood and Gee 1969, H. xenognathus Greenwood 1957, H. sp. 'green dentex', $H$. sp. cf. fusiformis [41-43], $H$. sp. cf. hiatus, $H$. sp. cf. 'supramacrops', $H$. sp. 'deepwater cinctus', $H$. sp. 'paropius like', $H$. sp. 'stone', and Platytaeniodus degeni Boulenger 1906. The fish were all collected by $\mathrm{M}$. A., S. M., and S. I. M. in 2004-2007. M. A. and S. M. verified all specimen identifications based on Additional file 6: additional text and Additional file 7: Table S2. Information for these species is provided in Additional file 1: Figure S1a-j. Four riverine species were also examined: $H$. sp. 'katonga', $H$. sp. 'katavi', $H$. sp. 'kitilda-rukwa', and $H$. sp. 'muzu-rukwa'. The localities for each riverine species are shown in Additional file 5: Figure S4.

\section{DNA sequencing and opsin sequence analyses}

Genomic DNA was extracted from caudal fins, pectoral fins, or muscular tissues using a DNeasy Blood \& Tissue kit (Qiagen, Hilden, Germany). All tissues were dissected and stored in $100 \%$ ethanol until use. Cichlid opsin genes (SWS1, SWS 2A, SWS2B, RH2A $\beta, R H 2 A \alpha, R H 2 B$, $L W S$, and $R H 1$ ) and mitochondrial DNA control region sequences were determined as described previously $[15,17,29,44]$. The downstream flanking sequences of LWS (Fig. 5a) were amplified by two rounds of PCR in a Takara PCR Thermal Cycler Dice Thermal (TakaraBio, Shiga, Japan). The first round amplified $3.5 \mathrm{kbp}$ of DNA fragments of downstream flanking using a pair of primers (LWSdown-longF and LWSdownlongR). The PCR program for the first round of amplification consisted of a denaturation step for $3 \mathrm{~min}$ at $94{ }^{\circ} \mathrm{C}$, followed by 30 cycles of denaturation for $1 \mathrm{~min}$ at $94{ }^{\circ} \mathrm{C}$, annealing for $1 \mathrm{~min}$ at $55^{\circ} \mathrm{C}$, and an extension for $3 \mathrm{~min}$ at $72{ }^{\circ} \mathrm{C}$. The second round of PCR used the products of the first PCR as templates, and the DNA fragments were amplified using a pair of primers (LWSB_F26 and LWSdown-longR). The PCR program for the second round of amplification consisted of a denaturation step for 3 min at $94{ }^{\circ} \mathrm{C}$, followed by 25 cycles of denaturation for $1 \mathrm{~min}$ at $94^{\circ} \mathrm{C}$, annealing for $1 \mathrm{~min}$ at $55^{\circ} \mathrm{C}$, and an extension for $2 \mathrm{~min}$ at $72{ }^{\circ} \mathrm{C}$. The PCR products were purified, and their sequences were determined by direct sequencing using an Applied Biosystems Automated 3130 Sequencer. The primers for determination of the sequence of PCR products were LWSB_F27, LWSB_F28, LWSB_R26, LWSB_R31, LWSB_R32, and LWSdown-longR. The positions and the sequences of primers were described by [29]. All sequences obtained were assembled with GENETYX version 10.0.1 or ATGC version 6.0.2 (Genetyx Corporation, Tokyo, Japan).

The sequences of eight opsin genes were determined from two individuals from each of the ten species, with the exception of the sequence for $R H 2 A \alpha$ from one individual Haplochromis sp. 'paropius like' $(n=1)$ and the sequence for $S W S 2 B$ from one individual $H$. sp. cf. fusiformis $(n=1)$. The mean Ds and Dn values were calculated using MEGA 5.05 [45]. FST values between species were calculated using mitochondrial DNA control region sequences by DnaSP 5.0 [46].

HKA (Hudson, Kreitman and Aguade) tests for heterogeneity were performed using the ratio of polymorphism to divergence [31] between regions: upstream of LWS (SWS2A gene: $1932 \mathrm{bp}$ ) and LWS gene (from exon 2 to exon $5,1286 \mathrm{bp}$ ), $L W S$ gene and downstream of LWS (1928 bp), and upstream (SWS2A) and downstream (Fig. 5a). To select the out-group among Lake Victoria LWS alleles for HKA tests, a LWS locus phylogenetic tree was constructed based on the sequences of the LWS gene and the flanking regions $(5 \mathrm{~kb}$ upstream, $L W S$ gene excluding exons, and $3.5 \mathrm{~kb}$ downstream, for a total of $9492 \mathrm{bp}$ ). The sequences of the LWS gene and the flanking regions from three and two individuals with $\mathrm{D}$ and Ds alleles, respectively, were determined by the method of [17]. The other sequences were obtained from previous studies $[17,18,29]$. The sequences were divided into alleles and concatenated to include the information of heterozygous sites. The tree was constructed using three methods (Neighbor Joining, Minimum Evolution, and Maximum Parsimony) calculated in MEGA 5.05 [45]. Based on this tree, the Sp allele was selected as the out-group for HKA tests (Fig. 5b).

\section{Reconstruction and measurement of visual pigment absorption spectra}

Production, reconstruction, purification, and measurement of the visual pigments were performed as described previously [17, 47], with minor modifications. Briefly, the sequences of $R H 1$ (rDs, rDb, rMga, and r217I alleles) were amplified by PCR using genomic DNA of Lake Victoria cichlids as a template with a pair 
of specific PCR primers [16] designed to produce a fusion protein with a FLAG-tag (Sigma-Aldrich; St. Louis, Missouri, United States) at the C terminus. The LWS-D and -Ds constructs were developed from the LWS-P construct [18] by PCR-based mutagenesis. The amplified DNA fragments were digested with restriction enzymes and cloned into the expression vector pMT5 [48] for LWS or pFLAG-CMV-5a (Sigma-Aldrich; St. Louis, Missouri, United States) for RH1. The visual pigments were reconstituted with A1- and A2-derived retinal, with A2derived retinal synthesized as described previously [49]. Absorption spectra of the pigment solutions in the presence of hydroxyl-amine $(<100 \mathrm{mM})$ before and after photobleaching were recorded using a spectrophotometer (UV-2400; Shimadzu; Kyoto, Japan), with 5-30 measurements before and after photobleaching. The mean peak spectral values (maximum absorption spectra: $\lambda \max$ ) and standard errors were determined from multiple preparations and measurements for each pigment. After reconstitution of the pigments, all procedures were performed under dim red or infrared light $(>900 \mathrm{~nm})$ using a digital video camera recorder (DCR-TRV8; Sony) in "night shot" mode or in complete darkness.

\section{Estimation of $\mathrm{A} 1 / \mathrm{A} 2$ ratio}

A photoreceptor cell includes both A1 and A2 pigments. MSP measures the absorption spectra of a single photoreceptor cell. The absorption spectra measured by MSP are, therefore, the sum of the absorption spectra of A1 and A2 pigments at a certain A1/A2 ratio. To estimate ratios of A1/A2 pigments in photoreceptor cells, LWS and RH1 standard absorption curves were first constructed using LWS and RH1 absorption curves (Fig. 4a-f) [29]. For each pigment (A1 and A2), the peaks of the curves ( $\lambda \max )$ of LWS and RH1 pigments were standardized, with the peak absorbance also standardized. To construct standard curves for A1 and A2 pigments, four absorption curves were averaged and then smoothed. The $\lambda \max$ of standard curves was adjusted to those of $L W S$ and RH1 alleles, with the standard absorption curves then used as template curves (the identical curves with different $\lambda \max$ ). For each $L W S$ allele, $\lambda \max$ of the mixture of $\mathrm{A} 1$ and $\mathrm{A} 2$ pigments with a changing A1/A2 ratio was estimated until it adjusted to MSP data. Absorption spectra of LWS pigments measured by MSP in Lake Victoria cichlid species were reported previously [20,33], and the A1/A2 ratio was estimated for these MSP data.

\section{Additional files}

Additional file 1: Figure S1. Short descriptions of the ten species and the frequencies of LWS and RHI alleles in the populations of (a) Haplochromis xenognathus, (b) H. sp. 'green dentex', (c) H. sp. 'paropius like', (d) Platytaeniodus degeni, (e) H. sp. 'stone', (f) H. piceatus, (g) H. sp. cf. hiatus, (h) H. sp. 'supramacrops', (i) H. sp. 'deepwater cinctus', and (j) H. sp. cf. fusiformis are shown in separate panels. Arabic numerals correspond to those in Fig. 1a, and the depths at each point are described on the right side of the numbers. The size of a pie indicates the number of haplotypes sequenced. The standard sizes of pies are shown at the left bottom. The colored sections of a pie indicate the frequency of the correspondent allele in the standard allele color pie (right bottom). The amino acid differences among allele groups are shown in Fig. 2b (LWS) and Fig. $2 \mathrm{C}(\mathrm{RH} 1)$. The maps were drawn by $\mathrm{Y}$. T. based on original source maps: https://www.google.com/maps. Fish photographs were taken by M. I. and S. M. (PDF 3 kb)

Additional file 2: Figure S2. Alignment of all polymorphic sites of LWS from the ten species. The nucleotide sites are shown on the top of the alignment. " $n$ " and " $s$ " indicate nonsynonymous and synonymous sites, respectively. Dots indicate the nucleotides that are identical with those in the top line. The allele groups of each sequence are shown on the right side of the sequences. (PDF $64 \mathrm{~kb}$ )

Additional file 3: Figure S3. An alignment of all polymorphic sites of RH1 from 10 species used in this study. The nucleotide sites are shown on top of the alignment. " $\mathrm{n}$ " and "s" indicate nonsynonymous and synonymous sites, respectively. Dots indicate where nucleotides are identical with those in the top line. The allele groups of each sequence are shown right side of the sequences. (PDF $62 \mathrm{~kb}$ )

Additional file 4: Table S1. $A 2$ ratio in $\mathrm{RH} 1$ and LWS pigments estimated from MSP data. (PDF 119 kb)

Additional file 5: Figure S4. Amino acid alignment of LWS from river species. Residue positions are numbered according to the sequences of LWS. The dots and letters indicate identical and different residues, respectively, compared with the top line. The LWS sequences were determined from four riverine species: H. sp. 'katonga' $(n=2), H$. sp. 'katavi' $(n=1), H$. sp. 'kitilda-rukwa' $(n=2)$, and H. sp. 'muzu-rukwa' $(n=3)$. (PDF $22 \mathrm{~kb})$

Additional file 6: Diagnosis and remarks of species used in this study. (DOCX $21 \mathrm{~kb}$ )

Additional file 7: General characters of Victoria cichlids used in the study. (XLSX $14 \mathrm{~kb}$ )

\section{Abbreviations}

LWS: Long-wavelength-sensitive opsin; $\mathrm{RH} 2$ : Middle-wavelength-sensitive opsin; SWS: Short-wavelength-sensitive opsin; $\lambda$ max: The peaks of the absorption curves

\section{Acknowledgments}

We thank Dr. Tetsu Sato (Graduate School of Bioscience and Biotechnology, Tokyo Institute of Technology, Japan; present address: Research Institute for Humanity and Nature, Kyoto Japan) and Tanzania Fisheries Research Institute (TAFIRI) for help with sampling.

\section{Funding}

This work was supported by JSPS KAKENHI Grant Number JP21227002 to N. O. and Grant Number JP23570269 and JP26440209 to Y. T., an internal SOKENDAI grant to Y. T., and a Center for the Promotion of Integrated Sciences (CPIS) of SOKENDAI grant to Y. T.

\section{Availability of data and materials}

The nucleotide sequences were deposited in GenBank under accession numbers LC129893-LC130491 and LC315405-LC315563. We deposited the phylogenetic data in TreeBASE (http://purl.org/phylo/treebase/phylows/ study/TB2:S21370).

\section{Authors' contributions}

YT: research concept, planning of research, DNA extraction, analyses of light environments, determination and analyses of opsin sequences,

measurements of opsin pigments, manuscript writing; RM: DNA extraction, determination of opsin sequences, opsin pigment measurements; MA: sampling, species identification; SM: sampling, species identification; HI: assistant for opsin pigment measurements; TO: synthesis of A2 retinal; AW: synthesis of $\mathrm{A} 2$ retinal; STK: determination of opsin and its flanking region 
sequences; AS: management of riverine Haplochromis species sample; HT: sampling and identification of riverine Haplochromis species; HDJM: sampling of Lake Victoria species; SIM: management and sampling of Lake Victoria species; NO: organization of research and sampling.

\section{Authors' information}

Not applicable.

\section{Ethics approval and consent to participate}

The Institutional Animal Care and Use Committee of Tokyo Institute of Technology approved the animal protocols and procedures. No human subjects were used in this study.

\section{Consent for publication}

Not applicable.

\section{Competing interests}

The authors declare that they have no competing interests.

\section{Publisher's Note}

Springer Nature remains neutral with regard to jurisdictional claims in published maps and institutional affiliations.

\section{Author details}

'Department of Evolutionary Studies of Biosystems, SOKENDAI (The Graduate University for Advanced Studies), Shonan Village, Hayama, Kanagawa 240-0193, Japan. ${ }^{2}$ Graduate School of Bioscience and Biotechnology, Tokyo Institute of Technology, 4259 Nagatsuta-cho, Midori-ku, Yokohama 226-8501, Japan. ${ }^{3}$ Department of Cellular and Molecular Biology, Primate Research Institute, Kyoto University, Kyoto, Japan. ${ }^{4}$ Department of Organic Chemistry for Life Science, Kobe Pharmaceutical University, 4-19-1, Motoyamakita-machi, Higashinada-ku, Kobe 658-8558, Japan. ${ }^{5}$ Department of Anatomy and Cytohistology, School of Dental Medicine, Tsurumi University, 2-1-3 Tsurumi, Tsurumi-ku, Yokohama 230-8501, Japan. ${ }^{6}$ Max-Planck-Institut für Biologie, Abteilung Immungenetik, Corrensstrasse 42, 72076 Tübingen, Germany. ${ }^{7}$ Tanzania Fisheries Research Institute (TAFIRI), Mwanza, Tanzania. ${ }^{8}$ Present address: Department of Life Sciences, National Cheng Kung University, 701 Tainan, Taiwan. ${ }^{9}$ Present address: Foundation for Advancement of International Science (FAIS), Tsukuba, Japan.

\section{Received: 28 March 2017 Accepted: 7 August 2017}

Published online: 22 August 2017

\section{References}

1. Seehausen O. Lake Victoria rock cichlids: taxonomy, ecology, and distribution. Zevenhuizen: Verduyn Cichlids; 1996.

2. Turner GF, Seehausen O, Knight ME, Allender CJ, Robinson RL. How many species of cichlid fishes are there in African lakes? Mol Ecol. 2001;10(3):793-806.

3. Johnson TC, Kelts K, Odada E. The holocene history of Lake Victoria. Ambio. 2000;29(1):2-11.

4. Nagl S, Tichy H, Mayer WE, Takahata N, Klein J. Persistence of neutral polymorphisms in Lake Victoria cichlid fish. Proc Natl Acad Sci. 1998;95(24): 14238-43.

5. Terai Y, Takezaki N, Mayer WE, Tichy H, Takahata N, Klein J, Okada N. Phylogenetic relationships among east African haplochromine fish as revealed by short interspersed elements (SINEs). J Mol Evol. 2004;58(1):64-78.

6. Samonte IE, Satta Y, Sato A, Tichy H, Takahata N, Klein J. Gene flow between species of Lake Victoria haplochromine fishes. Mol Biol Evol. 2007;24(9): 2069-80.

7. Wagner CE, Keller I, Wittwer S, Selz OM, Mwaiko S, Greuter L, Sivasundar A Seehausen O. Genome-wide RAD sequence data provide unprecedented resolution of species boundaries and relationships in the Lake Victoria cichlid adaptive radiation. Mol Ecol. 2013;22(3):787-98.

8. Terai Y, Mayer WE, Klein J, Tichy H, Okada N. The effect of selection on a long wavelength-sensitive (LWS) opsin gene of Lake Victoria cichlid fishes. Proc Natl Acad Sci U S A. 2002;99(24):15501-6

9. Shichida Y. Visual pigment: photochemistry and molecular evolution. In: Toyoda J, editor. The retinal basis of vision. Amsterdam: Elsevier Science; 1999.

10. Yokoyama S. Molecular evolution of vertebrate visual pigments. Prog Retin Eye Res. 2000;19(4):385-419.
11. Harosi FI. An analysis of two spectral properties of vertebrate visual pigments. Vis Res. 1994:34(11):1359-67.

12. Parry JW, Bowmaker JK. Visual pigment reconstitution in intact goldfish retina using synthetic retinaldehyde isomers. Vis Res. 2000:40(17):2241-7.

13. Carleton $\mathrm{KL}$, Harosi Fl, Kocher TD. Visual pigments of African cichlid fishes: evidence for ultraviolet vision from microspectrophotometry and DNA sequences. Vis Res. 2000;40(8):879-90.

14. Carleton $\mathrm{KL}$, Kocher TD. Cone opsin genes of african cichlid fishes: tuning spectral sensitivity by differential gene expression. Mol Biol Evol. 2001;18(8): 1540-50.

15. Sugawara T, Terai Y, Okada N. Natural selection of the rhodopsin gene during the adaptive radiation of east African Great Lakes cichlid fishes. Mol Biol Evol. 2002:19(10):1807-11.

16. Sugawara T, Terai Y, Imai H, Turner GF, Koblmuller S, Sturmbauer C, Shichida Y, Okada N. Parallelism of amino acid changes at the RH1 affecting spectral sensitivity among deep-water cichlids from lakes Tanganyika and Malawi. Proc Natl Acad Sci U S A. 2005:102(15):5448-53.

17. Terai Y, Seehausen O, Sasaki T, Takahashi K, Mizoiri S, Sugawara T, Sato T, Watanabe M, Konijnendijk N, Mrosso HD, et al. Divergent selection on opsins drives incipient speciation in Lake Victoria cichlids. PLoS Biol. 2006; 4(12):e433.

18. Seehausen O, Terai $Y$, Magalhaes IS, Carleton KL, Mrosso HD, Miyagi R, van der Sluijs I, Schneider MV, Maan ME, Tachida H, et al. Speciation through sensory drive in cichlid fish. Nature. 2008;455(7213):620-6.

19. Parry JW, Carleton KL, Spady T, Carboo A, Hunt DM, Bowmaker JK. Mix and match color vision: tuning spectral sensitivity by differential opsin gene expression in Lake Malawi cichlids. Curr Biol. 2005;15(19):1734-9.

20. Carleton KL, Parry JW, Bowmaker JK, Hunt DM, Seehausen O. Colour vision and speciation in Lake Victoria cichlids of the genus Pundamilia. Mol Ecol. 2005;14(14):4341-53.

21. Spady TC, Parry JW, Robinson PR, Hunt DM, Bowmaker JK, Carleton KL. Evolution of the cichlid visual palette through ontogenetic subfunctionalization of the opsin gene arrays. Mol Biol Evol. 2006;23(8):1538-47.

22. Hori M, Gashagaza MM, Nshombo M, Kawanabe H. Littoral fish communities in Lake Tanganyika: irreplaceable diversity supported by intricate interactions among species. Conserv Biol. 1993;7(3):657-66.

23. Kohda M, Hori M. Dichromatism in relation to the trophic biology of predatory cichlid fishes in Lake Tanganyika, East Africa. J Zool. 1993;229(3): 447-55.

24. Kawanabe H, Hori M, Nagoshi M. Fish communities in Lake Tanganyika. Kyoto: Kyoto University Press; 1997.

25. Fryer $\mathrm{G}$, lles TD. The cichlid fishes of the great lakes of Africa: their biology and evolution: Oliver and Boyd; 1972.

26. Seehausen O, Van Alphen JJ, Witte F. Cichlid fish diversity threatened by eutrophication that curbs sexual selection. Science. 1997;277(5333): 1808-11.

27. Seehausen $\mathrm{O}$, van Alphen JJ. The effect of male coloration on female mate choice in closely related Lake Victoria cichlids (Haplochromis Nyererei Complex). Behav Ecol Sociobiol. 1998:42(1):1-8.

28. Hofmann CM, O'Quin KE, Marshall NJ, Cronin TW, Seehausen O, Carleton KL. The eyes have it: regulatory and structural changes both underlie cichlid visual pigment diversity. PLoS Biol. 2009;7(12):e1000266.

29. Miyagi $R$, Terai Y, Aibara M, Sugawara T, Imai H, Tachida H, Mzighani SI, Okitsu T, Wada A, Okada N. Correlation between nuptial colors and visual sensitivities tuned by opsins leads to species richness in sympatric Lake Victoria cichlid fishes. Mol Biol Evol. 2012;29(11):3281-96.

30. Nagai $H$, Terai $Y$, Sugawara $T$, Imai $H$, Nishihara $H$, Hori M, Okada N. Reverse evolution in $\mathrm{RH} 1$ for adaptation of cichlids to water depth in Lake Tanganyika. Mol Biol Evol. 2011;28(6):1769-76.

31. Hudson RR, Kreitman M, Aguade M. A test of neutral molecular evolution based on nucleotide data. Genetics. 1987;116(1):153-9.

32. Maan ME, Hofker KD, van Alphen JJ, Seehausen O. Sensory drive in cichlid speciation. Am Nat. 2006;167(6):947-54

33. van der Meer HJ, Bowmaker JK. Interspecific variation of photoreceptors in four co-existing haplochromine cichlid fishes. Brain Behav Evol. 1995;45(4):232-40.

34. Bridges C, Yoshikami S. The rhodopsin-porphyropsin system in freshwater fishes-1. Effects of age and photic environment. Vis Res. 1970;10(12): 1315-IN1314.

35. Dalton BE, Loew ER, Cronin TW, Carleton KL. Spectral tuning by opsin coexpression in retinal regions that view different parts of the visual field. Proc Biol Sci. 2014;281(1797):20141980. 
36. Dalton BE, Lu J, Leips J, Cronin TW, Carleton KL. Variable light environments induce plastic spectral tuning by regional opsin coexpression in the African cichlid fish, Metriaclima Zebra. Mol Ecol. 2015;24(16):4193-204.

37. Maeda K, Takeda M, Kamiya K, Aibara M, Mzighani SI, Nishida M, Mizoiri S, Sato T, Terai Y, Okada N. Population structure of two closely related pelagic cichlids in Lake Victoria, Haplochromis pyrrhocephalus and $\mathrm{H}$. laparogramma. Gene. 2009;441(1):67-73.

38. Takeda M, Kusumi J, Mizoiri S, Aibara M, Mzighani SI, Sato T, Terai Y, Okada $\mathrm{N}$, Tachida $\mathrm{H}$. Genetic structure of pelagic and littoral cichlid fishes from Lake Victoria. PLoS One. 2013;8(9):e74088.

39. Takahashi T, Sota T. A robust phylogeny among major lineages of the east African cichlids. Mol Phylogenet Evol. 2016;100:234-42.

40. Toyama M, Hironaka M, Yamahama Y, Horiguchi H, Tsukada O, Uto N, Ueno $Y$, Tokunaga F, Seno K, Hariyama T. Presence of rhodopsin and porphyropsin in the eyes of 164 fishes, representing marine, diadromous, coastal and freshwater species-a qualitative and comparative study. Photochem Photobiol. 2008:84(4):996-1002.

41. Greenwood PH. A revision of the Lake Victoria Haplochromis species (Pisces, Cichlidae) Pt. II. H. sauvagei (Pfeffer), H. prodromus Trewavas, H. granli Blgr and H. xenognathus sp. n. Bull Brit Mus (Nat Hist) Zool. 1957;5:75-97.

42. Greenwood PH, Gee JM. A revision of the Lake Victoria Haplochromis species (Pisces, Cichlidae). Part VII. Bull Brit Mus (Nat Hist) Zool. 1969;18(1):1-65.

43. Boulenger GA. Descriptions of new fishes discovered by Mr. E. Degen in Lake Victoria. Ann Mag Nat Hist. 1906;17(7):433-52.

44. Mzighani SI, Nikaido M, Takeda M, Seehausen O, Budeba YL, Ngatunga BP, Katunzi EF, Aibara M, Mizoiri S, Sato T. Genetic variation and demographic history of the Haplochromis Laparogramma group of Lake Victoria-an analysis based on SINEs and mitochondrial DNA. Gene. 2010;450(1):39-47.

45. Tamura K, Peterson D, Peterson N, Stecher G, Nei M, Kumar S. MEGA5: molecular evolutionary genetics analysis using maximum likelihood, evolutionary distance, and maximum parsimony methods. Mol Biol Evol. 2011;28(10):2731-9.

46. Librado P, Rozas J. DnaSP v5: a software for comprehensive analysis of DNA polymorphism data. Bioinformatics. 2009;25(11):1451-2.

47. Ueyama H, Kuwayama S, Imai H, Tanabe S, Oda S, Nishida Y, Wada A, Shichida $Y$, Yamade $S$. Novel missense mutations in red/green opsin genes in congenital color-vision deficiencies. Biochem Biophys Res Commun. 2002; 294(2):205-9.

48. Khorana HG, Knox BE, Nasi E, Swanson R, Thompson DA. Expression of a bovine Rhodopsin gene in Xenopus Oocytes - demonstration of lightdependent ionic currents. P Natl Acad Sci USA. 1988;85(21):7917-21.

49. Wada A, Wang F, Ito M. A convenient and stereoselective synthesis of 11Z-3,4-Didehydroretinal by Horner-Emmons reaction using diphenyl phosphonate. Chem Pharm Bull. 2008:56(1):112-4.

\section{Submit your next manuscript to BioMed Central and we will help you at every step:}

- We accept pre-submission inquiries

- Our selector tool helps you to find the most relevant journal

- We provide round the clock customer support

- Convenient online submission

- Thorough peer review

- Inclusion in PubMed and all major indexing services

- Maximum visibility for your research

Submit your manuscript at www.biomedcentral.com/submit

) Biomed Central 